\title{
Parallel SOL flow on TCV
}

\author{
R. A. Pitts ${ }^{a, *}$, J. Horacek ${ }^{\text {a }}$, W. Fundamenski ${ }^{b}$, O. E. Garcia ${ }^{c}$, \\ A. H. Nielsen ${ }^{c}$, M. Wischmeier ${ }^{\mathrm{a}, 1}, \mathrm{~V}$. Naulin ${ }^{\mathrm{c}}$, \\ J. Juul Rasmussen ${ }^{\mathrm{c}}$ \\ ${ }^{a}$ Centre de Recherches en Physique des Plasmas, Association \\ EURATOM-Confédération Suisse, École Polytechnique Fédérale de Lausanne, \\ CH-1015 Switzerland \\ ${ }^{\mathrm{b}}$ Euratom/UKAEA Fusion Association, Culham Science Centre, Abingdon, OX14 \\ 3DB, United Kingdom \\ ${ }^{\mathrm{c}}$ Association EuratomRisøNational Laboratory, OPL-128 Risø, DK-4000 Roskilde, \\ Denmark
}

\begin{abstract}
Particle flow measurements parallel to the total magnetic field direction have been obtained for the first time in the TCV tokamak scrape-off layer. The plasma shape flexibility of TCV, coupled with carefully matched ohmic diverted discharges in forward and reversed toroidal field at varying plasma density is used to try and separate drift flow components and any field independent contributions in the outboard midplane vicinity. The measurements are generally well described in both direction and magnitude by neoclassical Pfirsch-Schülter return flows compensating poloidal drifts. There is clear evidence for a small, field independent offset component ( $\sim 10-20 \%$ of the main flow), whose magnitude would be approximately consistent with a contribution driven by enhanced outboard ballooning particle transport due to interchange motions, but a part of which could also be due to divertor sink action in the rather open TCV divertor configuration.
\end{abstract}

Key words:

PACS: 52.25.Xz, 52.55.Fa, 52.30.-q

JNM Keywords: P0500, P0600, T0100

PSI-17 Keywords: TCV, SOL transport, SOL plasma flow, Mach probe, Drifts

\footnotetext{
* Presenting and corresponding author: Email address: richard.pitts@epfl.ch (R. A. Pitts).

1 Present address: Institut für Plasmaphysik, Association EURATOM-IPP, Boltzmannstrasse 2, 85748 Garching, Germany
} 


\section{Introduction}

Particle flow parallel to the magnetic field in the scrape-off layer (SOL) is becoming increasingly suspected as an important mechanism contributing to tokamak material migration [1]. Reconciling the discrepancies between theory and experiment across machines and providing a predictive, fully consistent physics description of these SOL flows is an important challenge to plasma boundary research.

Strong parallel, co-current flow (from outer to inner targets) has been measured on JET near the top, low field side (LFS) of the poloidal cross-section [4], but with a magnitude that cannot be explained by parallel return "PfirschSchlüter" (P-S) flows which arise to compensate the non-divergence free poloidal $E_{r} \times \mathbf{B}$ and $\nabla \mathbf{p} \times \mathbf{B}$ classical drift flows in toroidal geometry [2]. Such P-S flows have previously been shown to be consistent with experiment in the outboard midplane region of JT-60U [3]. As this paper will show, a toroidal field $\left(B_{\phi}\right)$ independent offset has been observed on TCV, similar to recent observations on JET [4], both of which can be shown to be consistent with enhanced outboard radial particle transport due to turbulent interchange motions in the SOL [5]. Measurements of extremely strong inboard midplane parallel SOL flows on Alcator C-Mod have also been convincingly attributed to enhanced LFS perpendicular transport, whilst C-Mod outboard SOL flows can be described adequately by a combination of pure toroidal rotation and P-S flows $[6]$.

This contribution augments the tokamak SOL flow database by presenting a summary of the first parallel flow measurements on TCV,obtained in the outboard midplane region of single null lower (SNL) diverted discharges. Using matched pulses in forward (FWD- $B_{\phi}$, ion $\mathbf{B} \times \nabla \mathbf{B}$ drift downwards) and reversed $\left(\mathrm{REV}-B_{\phi}\right)$ toroidal field at varying plasma density, together with TCV's plasma shape flexibility, an attempt is made to separate classical flow components and any field independent contributions.

\section{Experiment}

Measurements are made using a fast reciprocating system ( $\mathrm{RCP}$ ) which inserts a 5 pin Langmuir probe head into the SOL plasma via a port located on the LFS vacuum vessel midplane. Figure 1 illustrates the probe trajectory into a standard SNL diverted equilibrium. Flux surface mapping from the probe location is used to express all radial profiles in terms of separatrix distances at the outboard midplane, where, henceforth in this article, "outboard midplane" should be taken to refer always to the LFS SOL region on the plasma magnetic 
axis and should not be confused with the vacuum vessel midplane.

The pins labelled 1 and 2 in the photographic insets of Fig. 1 are the field aligned Mach probe pairs, from which the parallel flow Mach number is derived from the ratio of ion saturation currents to the pins according to Hutchinson's theory [7]: $M_{\|}=v_{\|} / c_{s} \approx 0.4 \ln \left(I_{s a t, 1} / I_{s a t, 2}\right)$, where $c_{s}$ is the ion sound speed. Defined in this way, positive $M_{\|}$is directed from the outer to inner divertor targets along the magnetic field with negative values implying flow in the direction from the inner to outer targets. Each graphite probe pin measures $1.5 \mathrm{~mm}$ in length with diameter $1.5 \mathrm{~mm}$ and all are embedded in an electrically isolating boron nitride matrix secured in a graphite housing.

Although the RCP position on the torus is fixed, the shape flexibility offered by TCV allows the plasma to be displaced vertically such that different locations on the poloidal contour can be probed. To do so requires different probe heads arranged such that the pins match the poloidal contour of the magnetic equilibrium. Two different heads have been used thus far, both shown in Fig. 1. A wedge shaped head with $37^{\circ}$ angle, is used for standard SNL discharges in which the probe reciprocates through a region of expanded flux surfaces below the outer midplane. Profiles on the plasma midplane are obtained by lowering the plasma and using the flat probe head. In both cases, the poloidal array of 3 pins set in the central bar separating the Mach probe sensors is used to provide SOL profiles of $T_{e}, n_{e}$ and pin floating potential, $V_{f}$. The latter is used to estimate the plasma potential in the usual way, $V_{\text {plasma }} \sim V_{f}+2.8 T_{e}$ with $2.8 T_{e}$ being the probe sheath potential drop for a deuterium plasma [10].

Typically two probe reciprocations are made into each discharge and, in the data presented below, profiles from a number of probe scans, often in separate similar shots (labelled in the figures by shot group numbers), are combined and averaged. Experiments have been performed in ohmic plasmas at fixed plasma current, $I_{p}=260 \mathrm{kA}$. Only at this value or below can reproducible L-mode discharges be guaranteed in both FWD and REV- $B_{\phi}$ as a consequence of the lower ohmic L-H mode transition power threshold in FWD- $B_{\phi}$. Furthermore, $I_{p}$ is always reversed simultaneously with $B_{\phi}$ to preserve magnetic helicity and thus ensure that the Mach pins remain properly field aligned. On TCV, $\left|B_{\phi}\right|=1.43 \mathrm{~T}$ on the magnetic axis.

\section{Flow behaviour under toroidal field reversal}

Reversing $B_{\phi}$ whilst keeping all other discharge parameters as closely matched as possible should reveal the purely field dependent neoclassical parallel return flows compensating poloidal drifts. Figure 2a compiles the $M_{\|}$profiles resulting from this exercise in TCV for the SNL equilibrium of Fig. 1 over a line averaged 
density range of $\bar{n}_{e}=1.7 \rightarrow 7.4 \times 10^{19} \mathrm{~m}^{-3}$, corresponding to Greenwald fractions of $0.15 \rightarrow 0.45$. This latter value in fact corresponds to a density just below the density limit in this configuration. For both $B_{\phi}$ directions, $M_{\|}$ decreases strongly with $\bar{n}_{e}$ and reaches extremely high values $(\sim 0.6)$ at the lowest densities. For a given $B_{\phi}$ direction, the shape of $M_{\|}(r)$ bears some similarities to that observed near the outboard midplane of other divertor tokamaks $[6,8,3]$. At any given density, the general trends and magnitudes are also seen in Type III ELMing H-modes and in pure helium plasmas on TCV. Upon field reversal, for fixed $\bar{n}_{e}$ (except at the highest densities in the scan), the flows reverse, passing from net flow towards the outer divertor in REV$B_{\phi}$ (the normal field direction on TCV), to an inner divertor directed flow in FWD- $B_{\phi}$ and thus always in the co-current direction. These observations are also entirely consistent with the flow directions measured elsewhere.

If the total parallel flow is assumed to consist only of a field dependent drift component and a field independent transport contribution, the mean of the FWD- $B_{\phi}$ and REV- $B_{\phi}$ flows at each density should reveal any field independent offset, provided that SOL plasma profiles are well matched for each field direction and that geometry affects (such as magnetic field line connection to the probe) do not affect the measurements. Profiles of $n_{e}$ and $T_{e}$ are generally very similar for both directions of $B_{\phi}$ in Fig. 2a. Connection lengths in the main SOL are in the range of $20 \mathrm{~m}$ from the probe to inner and outer targets, much longer than the approximate probe collection length [9]: $L_{c o l}=d^{2} c_{s} / 8 D_{\perp} \sim 1.5$ $\mathrm{m}$ for typical SOL conditions during this density scan $(\mathrm{d}=15 \mathrm{~mm}$ is the probe housing dimension and a conservative cross-field particle diffusion coefficient, $D_{\perp}=1 \mathrm{~m}^{2} \mathrm{~s}^{-1}$ has been assumed). Values of $T_{e}$ measured by the upstream and downstream Mach pins are identical within experimental error. This would be expected if the parallel extent of the probe presheath is small in comparison with the probe to divertor target connection length.

Figure $2 \mathrm{~b}$ shows the mean flows computed from the FWD and REV- $B_{\phi}$ density pairs in Fig. 2a, revealing a negative offset in the range $M_{\|}=0.05 \rightarrow 0.1$ across the main SOL, with some evidence for an increase in magnitude at the highest density. The magnitude of this offset is in the range of 10-20\% of the main flow near the separatrix at low to medium densities. Whilst there is a tendency for a reproducible radial structure in these average profiles, the quality of the FWD- $B_{\phi} / \mathrm{REV}-B_{\phi} \mathrm{SOL} T_{e}, n_{e}$ profile match is probably insufficient to draw meaningful conclusions. What is clear, however, is that since the probe is located below the outer midplane for this configuration (in fact midway between the midplane and X-point), an excess flow towards the lower divertor would be consistent with enhanced outward transport in the midplane vicinity. 


\section{Poloidal distribution in the outboard midplane}

To investigate this flow offset further, an experiment has been performed in which the outer divertor leg of the SNLequilibrium in Fig. 1 is progressively shortened and the plasma lowered vertically in the vacuum vessel over three separate discharges. In this way, a limited scan of the parallel flow dependence on vertical position at the midplane is achieved. The vertical extent above and below the midplane $( \pm 10 \mathrm{~cm})$ to which this is possible is restricted only by the requirement that the poloidal plasma contour not depart too significantly from the planar geometry of the probe head that must be used in this case (see Fig. 1). Profiles of $M_{\|}$resulting from this experiment are shown in Fig.3a, where the insets provide the magnetic equilibria for the three discharges. This discharge series was achieved at only a single value of $\bar{n}_{e}$ and for REV- $B_{\phi}$ only. Profiles of $n_{e}, T_{e}$ and $V_{f}$ (parameters which determine the main contributions to the P-F flow) are extremely closely matched in the three discharges.

Throughout most of the SOL width, there is a clear decrease in the flow magnitude as the probe passes from below to above the outboard midplane. The incremental difference between each profile is of the same order as that found from the $B_{\phi}$ average in Fig.2b. Interestingly, of the three profiles, the one obtained precisely on the midplane is most similar in magnitude to the FWD$B_{\phi}$ profile at the same density $\left(\bar{n}_{e}=4.2 \times 10^{19} \mathrm{~m}^{-3}\right)$ shown in Fig. 2a for the standard SNL configuration in which the outer divertor leg is longest. For the probe position $10 \mathrm{~cm}$ below the midplane in Fig. 3, the measured flow is slightly stronger than obtained in Fig. 2a, where the probe is located $25 \mathrm{~cm}$ below the magnetic axis. The difference can be almost entirely prescribed to the SOL density (not shown) which is actually higher for the discharge in Fig. 2a than for those in Fig. 3. As a result, the Pfirsch-Schülter driven flow component (see below) is higher for the discharges in Fig. 3 and produces an increased flow towards the lower divertor (in REV- $B_{\phi}$ ). Differing SOL densities, despite the same $\bar{n}_{e}$, must be attributed to the configurational differences between the standard SNL and its vertically displaced counterparts.

The obvious interpretation of Fig. 3 is a poloidally dependent flow component which subtracts from the background drift flow (towards the outer target in this case) above the midplane and adds to it below. A clear candidate is a field independent transport driven flow which these results would indicate must be of order $M_{\|} \sim 0.05$, namely $\sim 10 \%$ of the background flow in the separatrix vicinity. The effect of any outer divertor sink action in these rather open divertor configurations cannot be excluded here, nor can it in the data of Fig. 2. This will be discussed further below. One important point worth noting, however, is that the probe to target connection length increases as the plasma vertical height increases (due to the lengthened outer divertor leg). 


\section{Explaining the flows}

It is evident from Figs. 2 that the dominant flow component near the TCV outboard midplane is: a) dependent on the direction of $B_{\phi}$ and directed against the $\mathbf{B} \times \nabla \mathbf{B}$ ion drift, b) highest at low $\bar{n}_{e}$ and $\mathrm{c}$ ) generally increases as the separatrix is approached. Pfirsch-Schülter return flows satisfy all of these criteria and are thus good candidates to explain much of these TCV observations. From a theoretical point of view, an expression for these P-S flows is unavailable for the general case of shaped plasmas such as those described here. A simple analytic approximation does exist for SOL P-S flows in the large aspect ratio, cylindrical approximation [10]:

$$
M_{\|}^{P S}=\frac{2 q \cos \theta}{c_{s}}\left(E_{r}-\frac{\nabla \mathbf{p}}{e n_{e}}\right) \frac{\mathbf{B}}{B^{2}}
$$

where $\mathrm{q}$ is the safety factor, $E_{r}$ and $\nabla p$ the radial electric field $\left(-d V_{\text {plasma }} / d r\right)$ and ion pressure gradient respectively and $\theta$ the poloidal angle, where $\theta=0$ is defined at the outboard midplane. Eqn. 1 ignores any pure poloidal $E_{r} \times \mathbf{B}$ drift not due to toroidal geometry. These flows reach maximum (and negative minimum) values at the outboard (inboard) midplanes respectively and are zero at top and bottom of the poloidal cross-section. Figure 4 compares the experimental flow data of Fig. 2 a with $M_{\|}^{P S}$ of Eqn. 1, where $T_{i}=T_{e}$ has been assumed and the data have been averaged and gradients estimated over a radial slice of width $4 \mathrm{~mm}$ in the main SOL centred on a separatrix distance of $10 \mathrm{~mm}$. Agreement is generally excellent across the entire range of $\bar{n}_{e}$ in both magnitude and direction between theory and experiment, demonstrating that these neoclassical return flows are responsible for the majority of the observed parallel flow in the outboard midplane vicinity.

Returning to the question of the small flow offset seen in Figs. 2 and 3, its possible origin is investigated in Fig. 5, which compiles the estimated flow profile at high density expected from two field independent contributions: a) flow driven by parallel temperature gradients arising as a consequence of the outer divertor target and b) flow driven by enhanced low field side cross-field particle transport ("ballooning"). The former has been estimated in TCV [11] using SOLPS5 (B2.5-Eirene) code simulations without drifts and in which the flow profile has been set arbitrarily to zero at the outermost flux surface of the simulation grid. In this case, flow is driven primarily by parallel $T_{i}$ gradients, which the code predicts are felt even above the $\mathrm{X}$-point in the rather open TCV divertor geometry.

The ballooning component is derived on the basis of simulations of the TCV outboard midplane SOL plasma using the 2D Electrostatic turbulence code ESEL which contains information only on magnetic field curvature and not 
direction. Statistical analysis of these simulations has recently been shown to provide remarkable agreement with identical analysis of density and flux turbulence measurements made with the same probe diagnostic and in the standard SNL configuration (though at slightly higher current, $I_{p}=340 \mathrm{kA}$ ) as that used here to obtain the data compiled in Fig. 2 [12]. This latter work demonstrates that fluid interchange motions must be responsible for the turbulent cross-field particle transport. A further step has been to associate these

"field aligned filaments" with bursts of parallel pressure whose effect is to drive a time-averaged flow at some fraction of a transient "sub-sonic" Mach number, assumed as $M_{\|}=0.5[5]$.

In the absence of the true parallel pressure gradient along the filament (ESEL currently simulates only in the 2D poloidal-radial domain), a simple Ansatz is adopted in which the time averaged, filament driven flow is given by: $\left\langle M_{\|}\right\rangle \approx 0.5 t(p>\alpha\langle p\rangle) / \Delta t$ where $t(p>\alpha\langle p\rangle) / \Delta t$ approximates the time over which the local pressure in any given event exceeds the time averaged value by some factor $\alpha$ ( $\Delta t$ is the total simulation time). This fractional time is computed across the radial simulation domain averaging the pressure in the poloidal direction to obtain $\langle p\rangle$. Results for 3 values of $\alpha$ are included in Fig. 5 for a simulation adapted to the highest density case in Fig. 2 (from [5]). The experimental FWD/REV- $B_{\phi}$ average flow at this density (Fig. 2b) has therefore also been included in Fig. 5 for comparison. Evidently, this simple approximation provides reasonable agreement with experiment, with higher values of $\alpha$ being favoured. However, drawing any quantitative comparison as to the appropriateness of any particular value of $\alpha$ given the approximate nature of the Ansatz employed must await the proper inclusion of parallel dynamics in ESEL, work which is underway.

\section{Conclusions}

Measurements of SOL particle flow parallel to the total magnetic field in the outboard midplane region of TCV diverted ohmic discharges clearly indicate a density and field direction dependence quantitatively consistent with neoclassical Pfirsch-Schülter return flows. Averaging of radial profiles obtained midway between the outboard midplane and X-point of matched SNL FWD and $\mathrm{REV}-B_{\phi}$ discharges reveals a field direction independent flow offset across the SOL width, with some indication for an increase in magnitude at the highest densities. A similar offset has been obtained in REV- $B_{\phi}$ when the plasma is displaced vertically such that flows are measured just above, below and at the midplane. Recent fluid turbulence simulations successfully reproducing turbulent statistics in the TCV SOL predict, using a simple Ansatz, a time averaged (and by definition field independent) flow which is in good qualitative agreement with the measured offset. Temperature gradients established as a 
result of the divertor target sink may also, however, drive a field independent

flow and further experiments and code simulations are required to eliminate this possibility.

\section{Acknowledgements}

This work was supported by the Swiss National Science Foundation, EURATOM, the UK Engineering and Physical Sciences Research Council and the Danish Center for Scientific Computing.

\section{References}

[1] R. A. Pitts et al., Plasma Phys. Control. Fusion 47 (2005) B303

[2] A. V. Chankin, J. Nucl. Mater. 241 - 243 (1997) 199

[3] N. Asakura et al., Phys. Rev. Lett. 84 (2000) 3093

[4] S. K. Erents et al., Plasma Phys. Control. Fusion 46 (2004) 1757

[5] W. Fundamenski et al., submitted to Nucl. Fusion

[6] B. LaBombard et al., Nucl. Fusion 44 (2004) 1047

[7] I. H. Hutchinson, Phys. Rev. A 37 (1988) 4358

[8] H. W. Müller et al., 32nd EPS Conference on Plasma Phys. Tarragona, 27 June - 1 July 2005 ECA Vol.29C, P-1.009 (2005)

[9] P. C. Stangeby, J. Phys. D 18 (1985) 1547

[10] P. C. Stangeby, (2000) The Plasma Boundary of Magnetic Fusion Devices, (Bristol, UK: Institute of Physics Publishing) ISBN 0750305592

[11] M. Wischmeier, Phd Thesis (No. TH 3176) École Polytechnique Fédérale de Lausanne (2005)

[12] O. E. Garcia et al., Plasma Phys. Control. Fusion 48 (2006) L1 


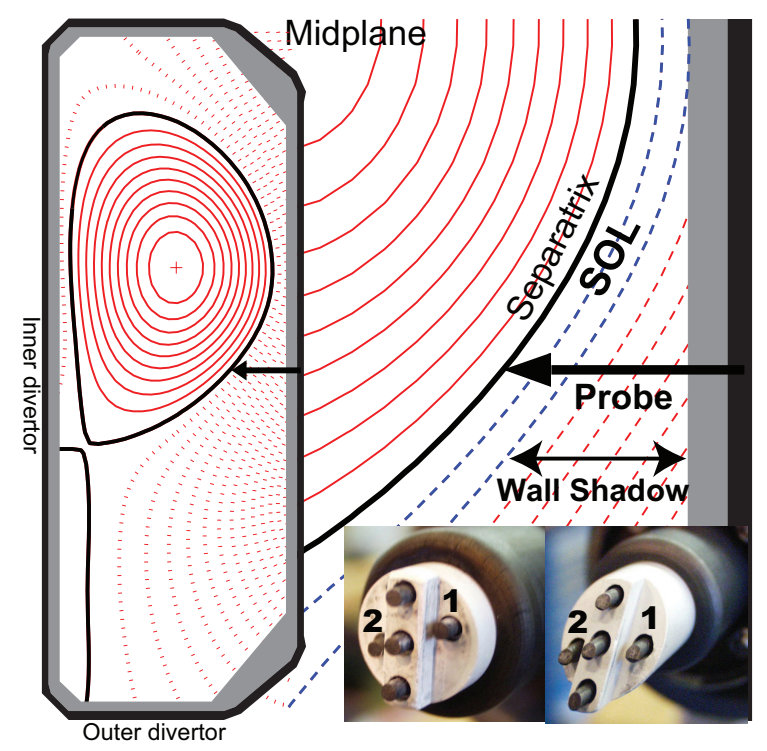

Fig. 1. Schematic illustrating the typical SNL discharge geometry, probe insertion location and Mach probe heads. 

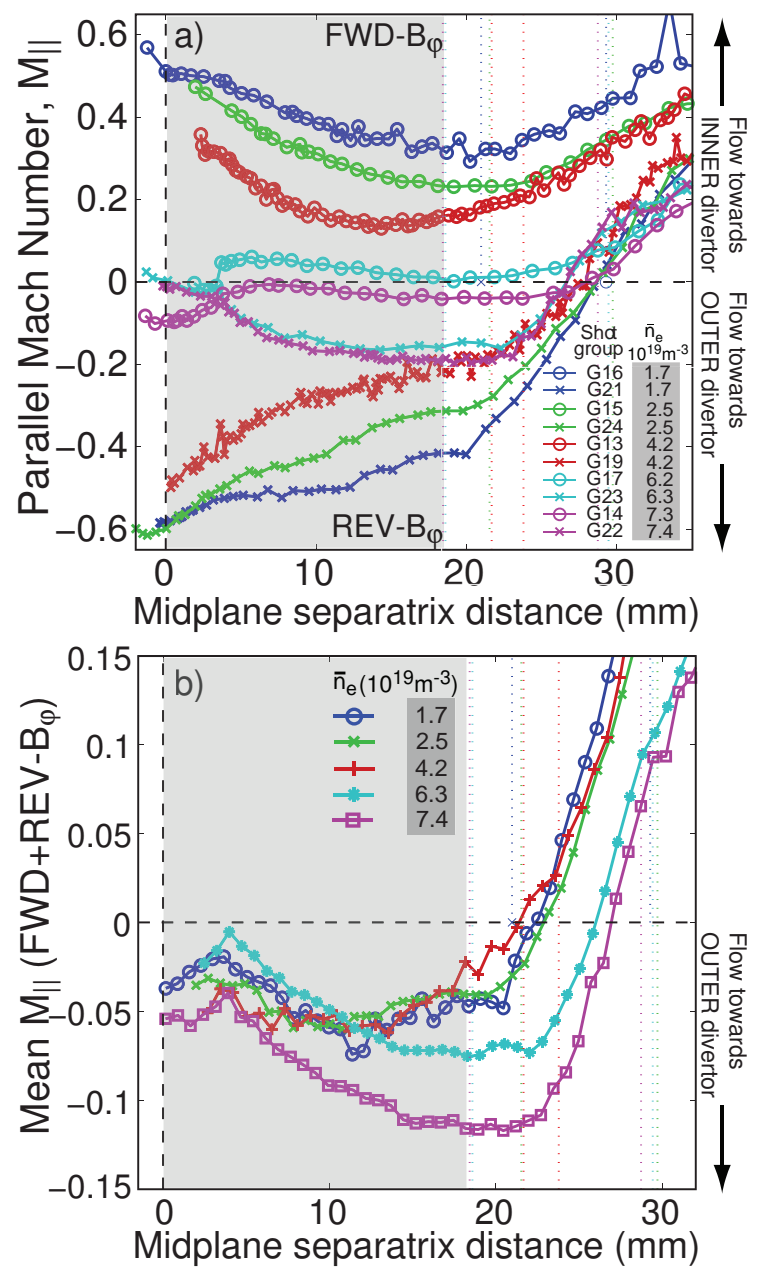

Fig. 2. a) Plasma flow below the outboard midplane in matched FWD- $B_{\phi}$ (open circles) and $\mathrm{REV}-B_{\phi}$ (crosses) plasmas at $I_{p}=260 \mathrm{kA}\left(q_{95} \sim 3.7\right)$. The vertical dashed lines delimit the outboard midplane wall radius (see Fig. 1), which varies from shot to shot, such that only in the shaded region, where field lines connect from inner to outer targets, can FWD- $B_{\phi}$ and REV- $B_{\phi}$ profiles be legitimately compared. Beyond this point, shorter connection lengths than in the main SOL drive strong flow modifications. b) Mean value of the FWD- $B_{\phi}$ and REV- $B_{\phi} M_{\|}$profiles in (a) 


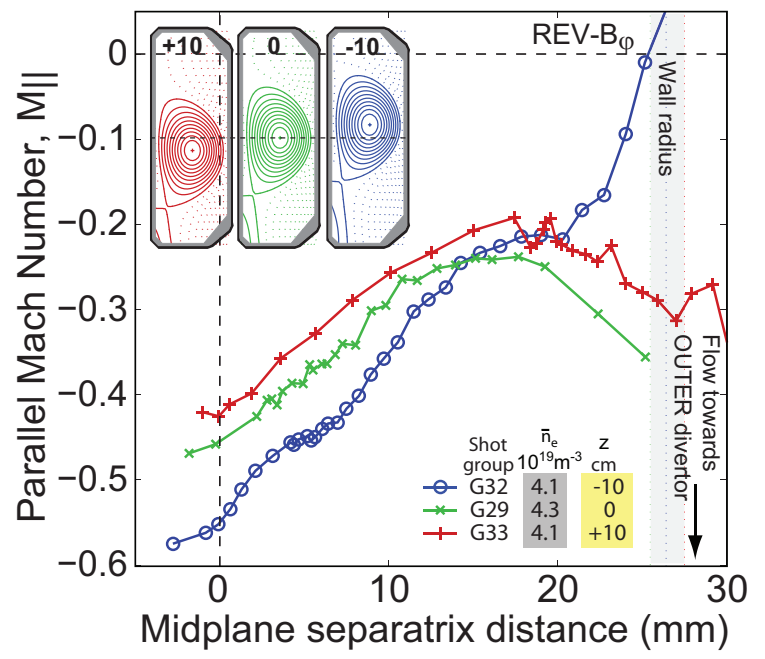

Fig. 3. Variation of REV- $B_{\phi}$ SOL flow with poloidal position in the outboard midplane vicinity at fixed density and $I_{p}=260 k A$. 


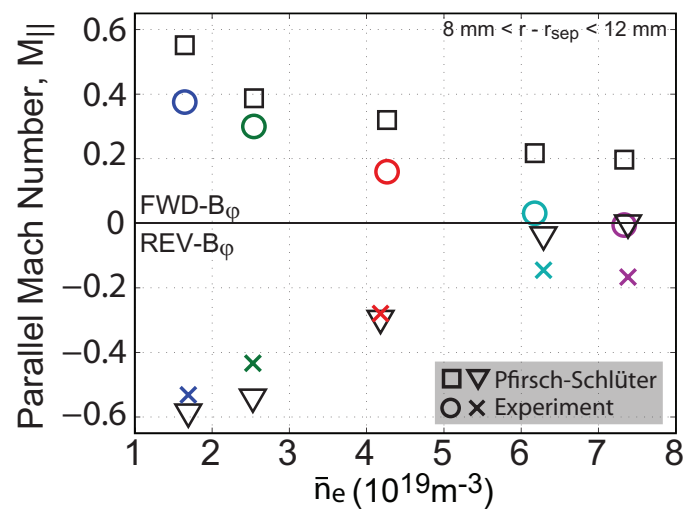

Fig. 4. Theoretical (Pfirsch-Schülter, Eqn. 1) and experimental Mach flows (Fig. 2a) compared for a $4 \mathrm{~mm}$ wide band of SOL plasma as a function of $\bar{n}_{e}$. Errorbars (not shown) on the calculated points are $\approx \pm 0.05$ 


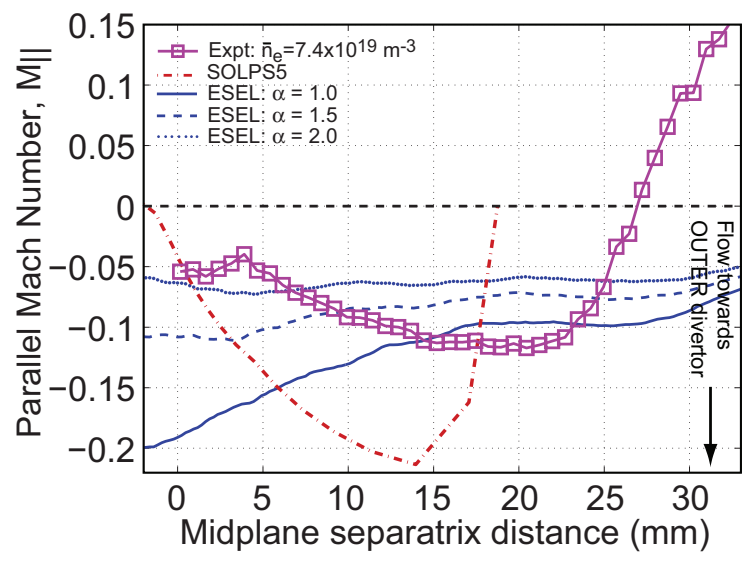

Fig. 5. High density FWD/REV- $B_{\phi}$ offset flow (Fig. 2b) compared with estimates of flow generation due to interchange turbulence near the outboard midplane (ESEL, [5]) and influence of the outboard divertor target (SOLPS5 [11]) 